\title{
Opening of an Enhancing Trainees' Learning Experiences through the Advanced Multiprofessional Simulation Training Facility at the University of Hertfordshire
}

\author{
Guillaume Alinier \\ Hertfordshire Intensive Care and Emergency Simulation Centre, University of Hertfordshire, \\ Hatfield, UK
}

\begin{abstract}
The University of Hertfordshire is currently setting up the largest medical simulation centre in UK and it is being presented in this article with some background information. This new centre includes a range of simulated clinical and community environments and makes use of modern patient simulators that will enhance the students' learning experience.
\end{abstract}

Key words: patient simulator; scenario-based training; simulation centre

\section{INTRODUCTION}

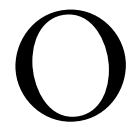
ver the years, the advancement of technology has enabled the commercialisation of ever more sophisticated and realistic training tools which can play a very important role in the acquisition of interpersonal, cognitive, and practical skills of future and currently practising healthcare professionals. Early simulation developments started in the 18th century

Correspondence to: Guillaume Alinier, Hertfordshire Intensive Care and Emergency Simulation Centre, University of Hertfordshire, College Lane, Hatfield, Hertfordshire AL10 9AB, UK. E-mail: G.Alinier@herts.ac.uk; Tel: +44 (0) 1707286395 through the work of the often forgotten Madame du Coudray, a French midwife, with her 'birthing machine' [Gelbart, 1998]. A century later another important model was made to help practise other life saving skills such as cardiopulmonary resuscitation using the Laerdal Resusci-Ann mannequins [Lind, 1961]. In parallel the first full-scale patient simulator was being developed [Abrahamson et al, 1969] but only to be ever used by a few privileged trainees. It is not until the 1990s that similar work culminated with the successful commercialisation of such simulators. Due to the price of these sophisticated interactive training models, their adoption has been somewhat slow but has now greatly improved thanks to the development of more affordable versions primarily relying on an operator to modify the physiological parameters according to the treatment provided by trainees and the desired patient case to be simulated. It is estimated that over 4500 medium- to high-fidelity patient simulators have been sold around the world [AIMS, 2007], approximately $10 \%$ of which are in the UK. While they have still mostly been adopted by western countries, several developing countries are currently trying to set up simulation programmes. The University of Hertfordshire is among the early adopters of studentlead scenario-based simulation training in the UK with the opening of its Hertfordshire Intensive Care and Emergency Simulation Centre (HICESC) in 1998. The centre was initially a simulated clinical environment primarily used by nursing and paramedic students and evolved over time as new and more affordable simulation technology became available and their use in addition to traditional training sessions was more widely accepted. 


\section{DEVELOPMENT OF OUR MEDICAL SIMULATION CENTRE}

HICESC has been through four major developmental phases which can be described as:

1: Demonstration ward (1998-2001)

2: All-in-one simulation room (2001-2004)

3: Dedicated simulation room with adjacent observation room (2004-2006)

4: Multi-simulation rooms with observation rooms (since 2006)

The centre originated from a collaborative venture between the Schools of Nursing and Paramedic Sciences and Electronic, Communication and Electrical Engineering. During the first two phases, the centre was located in a biomedical engineering laboratory and was equipped as an ICU with three adult bays, an incubator and a paediatric bed. Until the arrival of Laerdal SimMan patient simulators in 2001, the beds were occupied by Laerdal ALS mannequins which allowed for basic scenarios to be practised. In 2000 the HICESC steering committee was awarded a grant from the BHF Foundation which enabled the centre to develop expertise in organising OSCEs [Alinier, 2003] and running simulation sessions [Alinier et al, 2006a]. This helped the centre moving away from running practical sessions where the tutor was simply demonstrating or prompting students. In the second phase partitions were used to divide the room in three areas: the simulation area, where the students were taking care of the patient simulator; the observation area, which had an audio/video $(\mathrm{A} / \mathrm{V})$ link from the simulation area for other students to observe their peers in an unobtrusive manner; and the control desk, for an operator to discretely control the physiological parameters and the voice of the patient simulator (Fig. 1). Although this setup was functional it had limitations such as the observers having to remain silent during the scenarios in order not to distract their peers. During that phase several pilot simulation sessions were run to test different approaches, levels of scenario difficulty, duration of sessions and scenarios, and number of participants.

By the third phase, HICESC had become a real asset for the Faculty of Health and Human Sciences and it was relocated to another laboratory with an adjacent room which was used as the observation and debriefing room (Fig. 2). The setup of the simulation area with its small partitioned control desk was virtually the same as in the previous phase. The improvements were the observation room with its $\mathrm{A} / \mathrm{V}$ link and repeated patient monitor, and the raised floor which simplified the connectivity of the patient simulators and $\mathrm{A} / \mathrm{V}$ equipment.

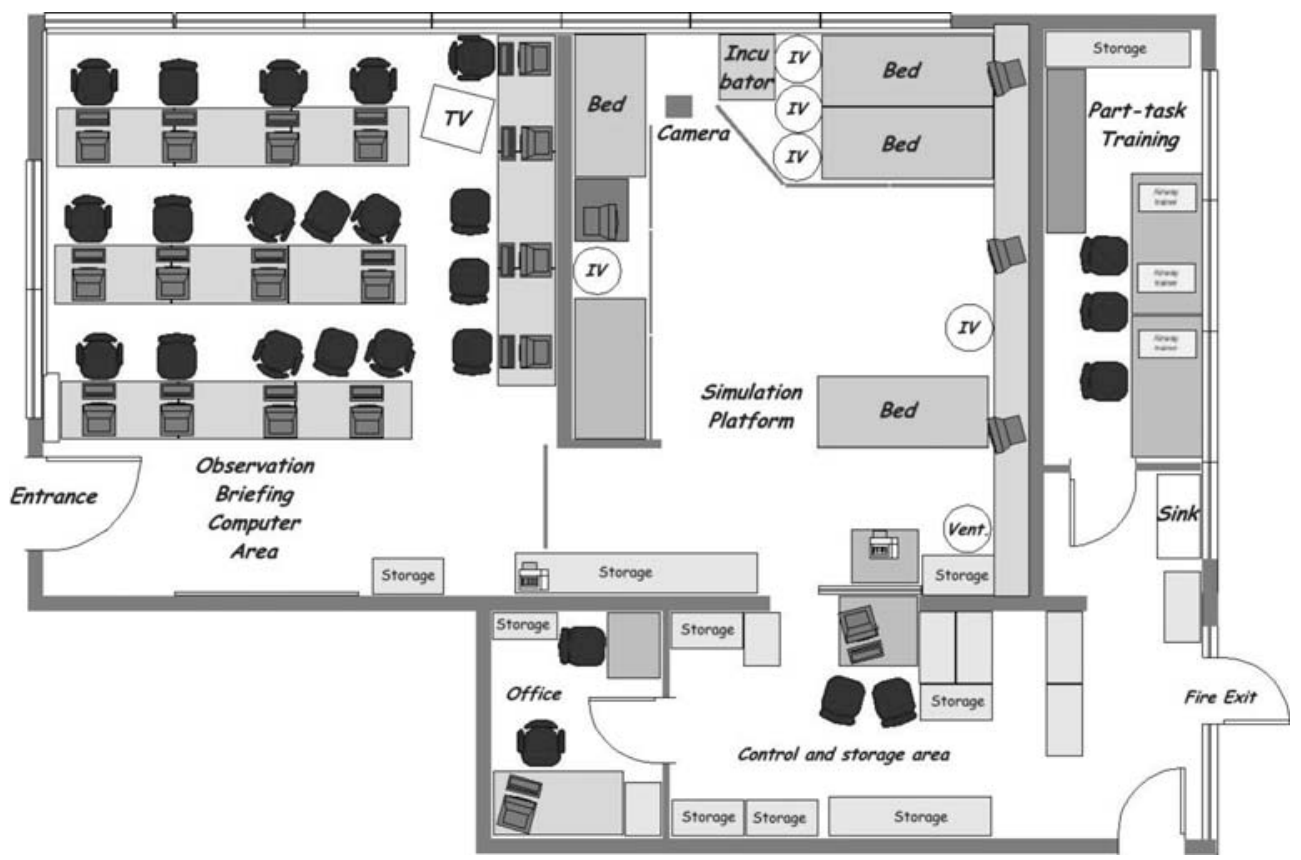

Figure 1. HICESC as a 'all-in-one' simulation facility with mobile partitions and configured for a simulation session. 


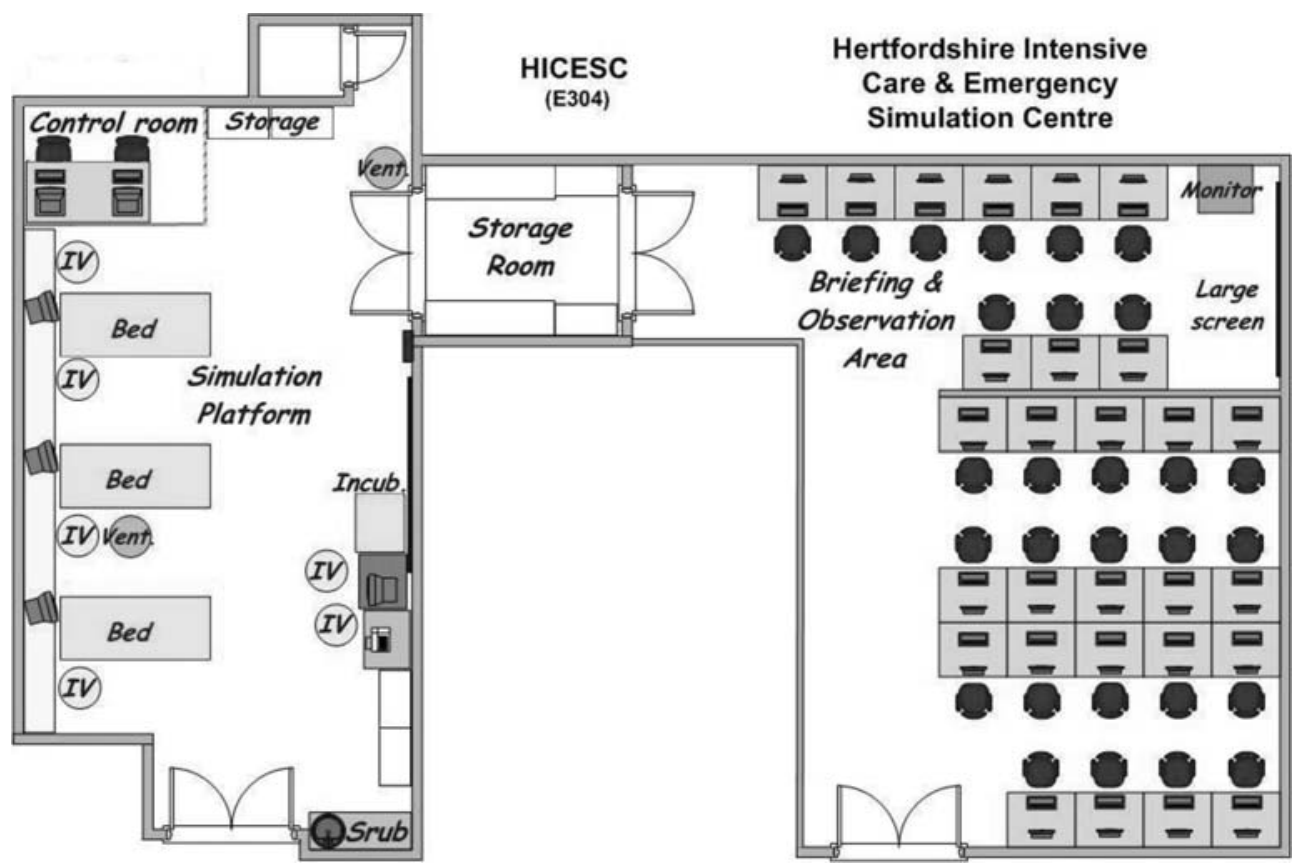

Figure 2. HICESC with a separate but shared observation/debriefing room in an adjacent computer laboratory.

The main disadvantage was that the observation room was shared with another school, hence putting limitations to the number of sessions that could be run each week. During that stage HICESC was increasingly used, not only by university students but also by external candidates and companies for short courses. This encouraged the development of a totally new facility with a multidisciplinary focus.

\section{MULTIPROFESSIONAL MEDICAL SIMULATION TRAINING CENTRE}

Now in its fourth phase of development, HICESC is housed in a purpose built facility which is currently the largest medical simulation centre in the UK (Fig. 3). The centre has an adult and a paediatric ICU which can both be set up as operating theatres, a recovery or general ward with four bays which can also be used as an A\&E, a household setting with bedroom, dining room and bathroom, a GP patient assessment room, and a 16 computer workstation simulation facility. One of the unique features of the centre is that it also houses a fully stocked-up community and hospital pharmacy with an automated dispensing robot, a customer counter, a needle exchange counter, a counselling room, and a preparation area. Instrumental to the operations of the centre are two control rooms to operate the patient simulators and control the $\mathrm{A} / \mathrm{V}$ system, three observation/debriefing rooms that can open up as one large conference room and storage facilities. The building benefits from a large bay by the front entrance to park an ambulance. The centre has been designed as a flexible learning environment with mobile walls between some of the rooms and networked monitors to display a timer when used for large scale practical tests such as OSCEs. It is equipped with $26 \mathrm{pan} / \mathrm{tilt} / \mathrm{zoom}$ digital video cameras and 8 patient monitors video feeds, all of which can be recorded and projected in any of the observation rooms or any computer with an internet connection.

Such a development comes at a cost which was not covered by a major grant but thanks to many who raised money through fundraising and collaboration with industrial partners such as Eisai, Scotia, and charitable foundations. Due to the specialised activity of the centre, this project was very complex even for architects and contractors with experience of the hospital building sector. For example, the setup allows to recreate medical gases and power failures, has a controllable and bidirectional communication system between all rooms, phone lines, and mobile suspended cameras. Due to the computing and video technicality of the equipment in the centre, it is staffed by a small team of academic and technical simulation specialists and supported by tutors from different disciplines when students come to take part in simulation sessions. 


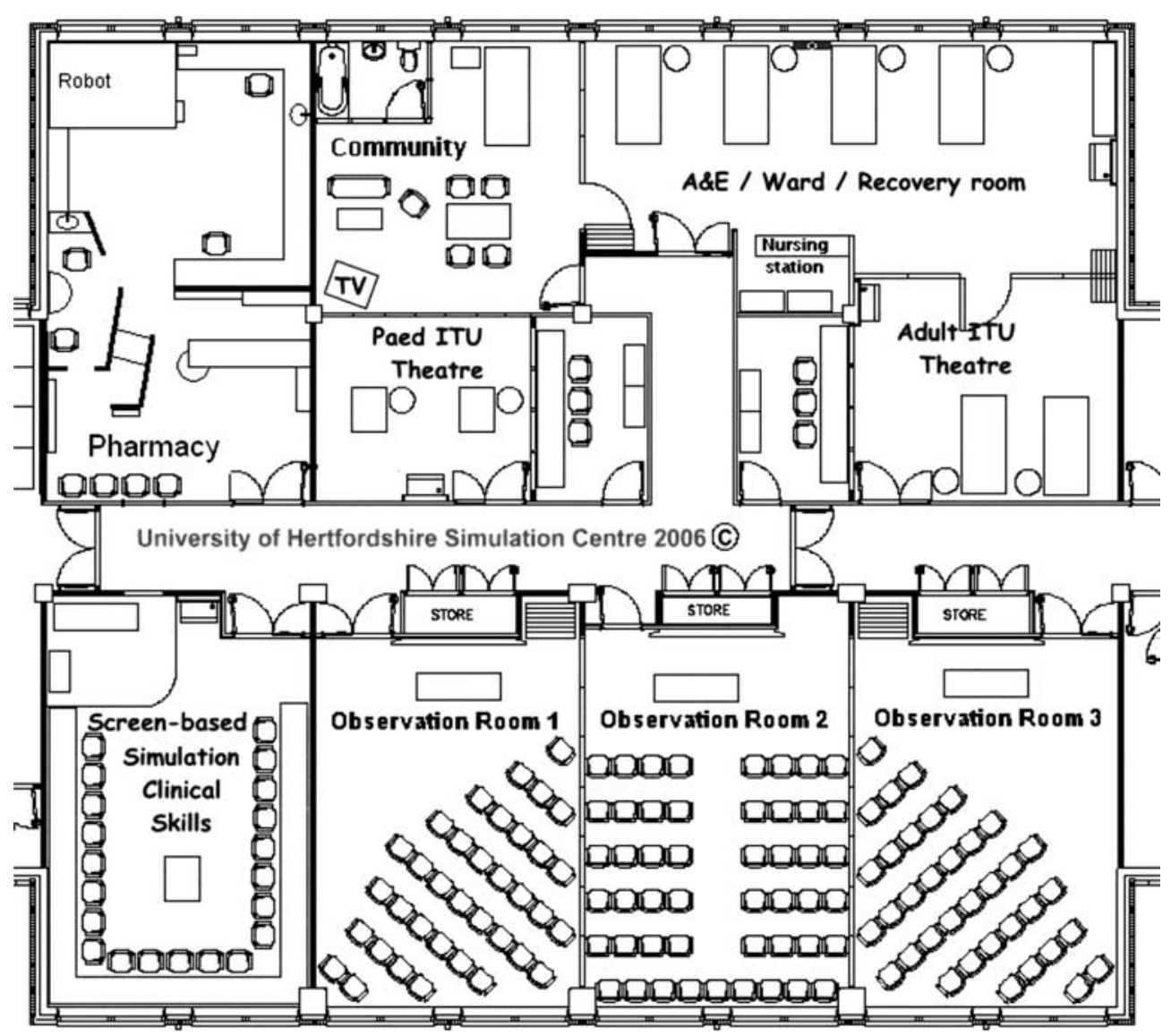

Figure 3. Current HICESC facilities as a large multiprofessional simulation centre.

This new centre provides unique opportunities for students to follow and critically analyse the patient journey from arrival in A\&E to home discharge when they are observers. Students who are actually taking part in a scenario can be charged with the responsibility of the 'patient' care to gain experience as they are working in a safe and controlled environment as can be observed in Fig. 4. This type of approach is very favoured by students as they are perpetually challenged by the evolving condition of the patient simulator and have to 'think on their feet'. Students only access the facility for scenario-based simulation training when they have acquired a certain level of knowledge and skills that they will have had the opportunity to practise uniprofessionally in one of the clinical skills laboratories. The different environments of this new simulation centre lend themselves to running interprofessional simulation sessions which enhances the realism of the scenarios, and help students better understand other healthcare professional roles and develop communication and teamwork skills. A Higher Education Academy Health Sciences and Practice Subject Centre Funded Project is currently taking place in HICESC with final year healthcare students to research this very issue.

\section{EQUIPMENT AND ACTIVITIES}

The centre is currently occupied by two Laerdal SimMan, a SimBaby, nursing Kelly, nursing Anne (Both with Vital signs), junior, and other adult and paediatric mannequins. There is also a wide range of medical equipment, some of which have been donated by local NHS Trusts (disused operating table, anaesthetic machine) or on loan from companies (i.e. Datascope, Laerdal Medical, Alaris Medical, Welch Allyn, Huntleigh Healthcare, Bristol Maid, Lidco ...) which make the different environment very realistic. The centre 


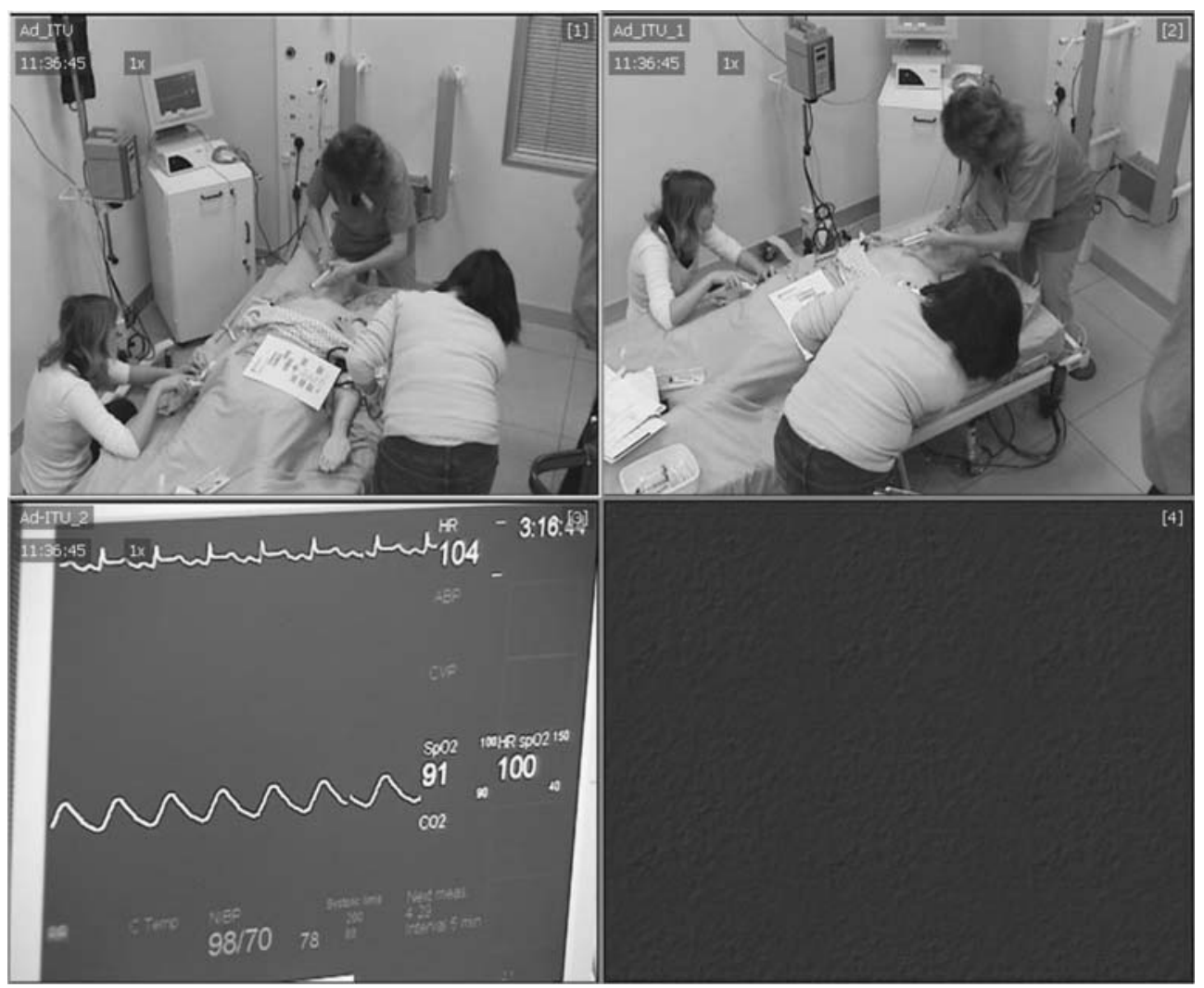

Figure 4. View of the screen displayed in the observation during a simulation session with a group of junior doctors.

also makes use of simulated patients, who are volunteers, for some of the scenarios or OSCE stations.

In terms of activities, the centre is used for simulation training by schools from the Faculty of Health and Human Sciences as well as by external organisations and institutions. There is an ongoing programme of short courses, some of which are specifically targeting new simulation facilitators or technicians to train them to design and run simulation sessions, programme scenarios, or control the patient simulator. It is recognised and recently discussed by Issenberg [2006] that tutors, irrespective of experience and seniority, need training to understand the simulation training philosophy making use of interactive technology. Realistic simulation requires an approach different to any other traditional teaching methods and requires specific training. Other courses are for Continuing Professional Development of qualified healthcare professionals and include Advanced Medical Life Support (AMLS) and custom designed scenario-based simulation training sessions that may focus on crisis resource management [Holzman et al, 1995] in any clinical setting.
The centre is regularly engaged with the wider simulation community to share and benefit from others' experience. These mainly include the UK National Association for Medical Simulation (NAMS), the Society in Europe for Simulation Applied to Medicine (SESAM), and the Society for Simulation in Healthcare (SSH), which have annual meetings. Our commitment to collaborate with others will shortly be marked by two conferences hosted by HICESC in the near future [NAMS, 2007; SESAM, 2008]. Staff are constantly engaged in research projects [Alinier et al, 2006b] and consultancy activities relating to medical simulation.

\section{CONCLUSION}

It is fairly well recognised that 'practice makes perfect', so exposing students to simulated critical incidents getting them to respond in a realistic manner, followed up with a debriefing session encouraging students to reflect can only be beneficial. Should a similar event happen 
for real, they should be more prepared in recognising it and providing the best care. The actual transferability of skills acquired by candidates to real practice is unfortunately often only supported by anecdotal evidences and might be biased by their enthusiasm and excitement for using modern technology.

Although it has taken a long time, the different stages of development of HICESC have allowed us to eventually design a very practical facility which benefits from many customised features. The clinical skills training laboratories have deliberately remained a separate entity from the simulation centre in order not to duplicate resources or dilute the simulation activities.

Our commitment to improve the teaching and learning experience of students has driven this innovative development as we strongly believe that simulation training will help students acquire some valuable experience. We aim to offer students within the Faculty of Health and Human Sciences and the regional healthcare workforce a first class training facility where they can learn alongside each other, acquire new skills or knowledge, and reflect, to become better and safer professionals.

\section{REFERENCES}

Abrahamson S, Denson JS, Wolf RM. Effectiveness of a simulator in training anesthesiology residents. Journal of Medical Education 1969; 44(6): 515-519.

Advanced Initiatives in Medical Simulation (AIMS). http:// www.medsim.org/coucil.asp accessed on $11 / 04 / 07$.

Alinier G. Nursing students' and lecturers' perspectives of OSCE incorporating simulation. Nurse Education Today 2003; 23(6): 419-426.

Alinier G, Hunt B, Gordon R, Harwood C. Effectiveness of intermediatefidelity simulation training technology in undergraduate nursing education. Journal of Advanced Nursing 2006a; 54(3): 359-369.
Alinier G, Gordon R, Harwood C, Hunt B. 12-Lead ECG training: the way forward. Nurse Education Today 2006b; 26(1): 87-92.

Gelbart NR. The King's Midwife: A History and Mystery of Madame du Coudray. Berkeley and London: University of California Press, 1998.

Holzman RS, Cooper JB, Gaba DM, Philip JM, Small SD, Feinstein D. Anesthesia crisis resource management: real-life simulation training in operating room crises. Journal of Clinical Anesthesia 1995; 7: 675-687.

Issenberg SB. The scope of simulationbased healthcare education. Simulation in Healthcare 2006; 1 : 203-208.
Lind B. Teaching mouth-to-mouth resuscitation in primary schools. Acta Anaesthesiologica Scandinavica 1961; 9 (Suppl): 63-69.

National Association of Medical Simulators UK (NAMS). http:// www.namsonline.com accessed on 18/05/2007.

Society in Europe for Simulation Applied to Medicine (SESAM). http://www.sesam.ws accessed on 18/05/2007.

Society for Simulation in Healthcare (SSH). http://www.ssih.org accessed on $18 / 05 / 2007$. 